\title{
Identification of human balance control in standing ${ }^{*}$
}

\author{
Herman van der Kooij \\ Biomechanical Engineering, BMTI \\ University of Twente, \\ P.O. Box 217, 7500 AE Enschede, The Netherlands \\ h.vanderkooij@ctw.utwente.nl
}

\author{
Stella Donker, Maaike de Vrijer, Frans van der \\ Helm \\ Biomechanical Engineering, BMTI \\ University of Twente, \\ P.O. Box 217, 7500 AE Enschede, The Netherlands
}

\begin{abstract}
The goal was to identify the contribution of intrinsic mechanical properties of the muscular-skeletal system and the reflex gains in controlling balance during standing. The combination of balance perturbations experiments and closed loop identification schemes made it possible to identify the reflex loop gains and intrinsic mechanical properties in various environmental conditions. Human balance responses were studied by placing subjects on a movable support base while keeping their eyes open or closed. EMG, body motion and the ground reaction forces were recorded. From the platform perturbation data the Frequency Response Functions of the controller dynamics and the admittance of the balance control system were estimated with a non-parametric closed loop identification technique. Using a parametric model of balance control and a fitting procedure the sum of the intrinsic stiffness and neural position feedback gain, the neural time delay, the neural velocity feedback gain and the intrinsic damping were uniquely identified. The results show that subjects balancing on a randomly moving platform in the forward-backward direction applied a minimal stiffness strategy. The required damping to avoid oscillations was mainly due to neural velocity feedback rather than to intrinsic damping
\end{abstract}

Keywords: Balance control, identification, reflex loop.

\section{Introduction}

The keen interest into human balance control has resulted in many publications in which the common goal was to elucidate the mechanisms of balance control. The impedance of the ankle joint is a particular topic of interest. Many researchers tried to obtain the ankle impedance from experimental data using almost as many different methods. One of the major goals in studying human balance control is to identify the contribution and nature of intrinsic and reflexive feedback pathways in controlling balance in various environmental conditions. The intrinsic feedback have been modeled as intrinsic stiffness $\left(K_{i}\right)$ and damping $\left(D_{i}\right)$ due to muscle mechanics and the mechanical properties of connective tissues. The reflexive feedback pathways has been modeled as neural position $\left(K_{r}\right)$ and velocity $\left(D_{r}\right)$ feedback gains with a neural time delay $(\tau)$ in the feedback loop. The quasi stiffness obtained from the linear regression of joint angles and moments has also been used as a measure of ankle stiffness, although this measure is erroneous [2]. The results of many attempts to estimate the impedance of the ankle joint are ambivalent since they are based on the

Table 1

Overview of estimated intrinsic stiffness $\left(K_{i}\right)$ and damping $\left(D_{i}\right)$, proportional $\left(K_{r}\right)$ and velocity $\left(D_{r}\right)$ neural feedback gains and quasi stiffness obtained from linear regression of joint moment and joint angle time series. SB denotes the conditions in which the support base was rotated and VIS denotes the conditions in which the visual surround was rotated. In [1] the time series were segmented into five segments from slow to fast movements. For each segment the quasi stiffness was estimated.

\begin{tabular}{|c|c|c|c|c|c|}
\hline Ref & $K_{i}[\mathrm{Nm} / \mathrm{rad}]$ & $D_{i}[\mathrm{Nms} / \mathrm{rad}]$ & $K_{r}[\mathrm{Nm} / \mathrm{rad}]$ & $D_{\mathrm{r}}[\mathrm{Nms} / \mathrm{rad}]$ & Quasi stiffness [ $\mathrm{Nm} / \mathrm{rad}]$ \\
\hline [1] & & & & & $\begin{array}{l}17 \mathrm{e} 2(0.2 \mathrm{~Hz}) \\
17 \mathrm{e} 2(0.4 \mathrm{~Hz}) \\
20 \mathrm{e} 2(0.6 \mathrm{~Hz}) \\
26 \mathrm{e} 2(0.85 \mathrm{~Hz}) \\
46 \mathrm{e} 2(1.25 \mathrm{~Hz})\end{array}$ \\
\hline [3] & 572.600 & $5.6-11.4$ & & & \\
\hline [4] & $485 \pm 242$ & & & & \\
\hline$[5]$ & $824 \pm 128$ & $2.8 \pm 0.32$ & & & \\
\hline [7] & & & & & $1030-1374$ \\
\hline$[8]$ & $\begin{array}{l}91 \pm 29 \text { (SB) } \\
120 \pm 26 \text { (VIS) }\end{array}$ & $\begin{array}{l}25 \text { (SB) } \\
189 \text { (VIS) }\end{array}$ & $\begin{array}{l}968(\mathrm{SB}) \\
976 \text { (VIS) }\end{array}$ & $\begin{array}{l}332(\mathrm{SB}) \\
286(\mathrm{VIS})\end{array}$ & \\
\hline
\end{tabular}

* 0-7803-8566-7/04/\$20.00 @ 2004 IEEE. 
erroneous application of open-loop identification techniques to balance control, which is a closed-loop system[2]. Without claiming to be complete some reported values are given (Table 1). The differences in the reported values are apparent. In this paper the closed-loop identification schemes derived in [2] were applied to estimate the Frequency Response Functions (FRF's) of the admittance and control dynamics of human balance obtained from support base perturbation experiments. The FRF's of a parametric model of balance control are fitted to the estimated FRF's. The model fit results in estimates of $K_{i}, D_{i}, K_{r} D_{r}$ and $\tau$.

\section{Methods}

\subsection{Experiments.}

\section{Participants}

Four women and five men (mean age $=26$ years; range $=23$ to 33 years, mean body mass $=74 \mathrm{~kg}$, range $61-$ $84 \mathrm{~kg}$, mean body length $=1.82 \mathrm{~m}$, range $1.68-1.93 \mathrm{~m}$ ) participated voluntarily in the experiment. All participants were healthy and did not suffer from any motor impairments or movement-related disorders. All participants gave their written informed consent prior to the start of the experiment.

\section{Apparatus(CAREN, Motek Inc) and Recordings}

The participants stood barefoot on a computer controlled 6DOF motion platform with their feet slightly separated (Fig. 1). They faced a visual scene $(4.80 \times$ $3.70 \mathrm{~m}$ at a distance of $3.20 \mathrm{~m}$ ) consisting of a light grey background on which randomly distributed black dots with diameters varying from 10 to $80 \mathrm{~cm}$ were depicted. At eye level a number was projected which served as a focus point. Except from the light from the scene and the infrared light emitted by the movement registration cameras, the room was dark.

Displacementsof heel, toe, malleolus, tibia, knee, femur, iliac crest of both legs as well as the displacement of the sacrum, head and shoulder were determined using reflective spherical markers. Moreover, markers were attached to the motion platform to record the actual displacements of the platform. The positions of the markers were recorded at a sampling rate of $120 \mathrm{~Hz}$ by means of a 3D motion capturing system (VICON), consisting of six video cameras and a control unit. Body weight and length were measured for each participant. The joint angles, angular velocities and segment energies and the Center of Mass (CoM) of the whole body relatively to the ankle joint were calculated following the procedures described by .[6]. EMG was recorded with a sample frequency of $1024 \mathrm{~Hz}$. Ground reaction forces were recorded with an AMTI force plate with a sample

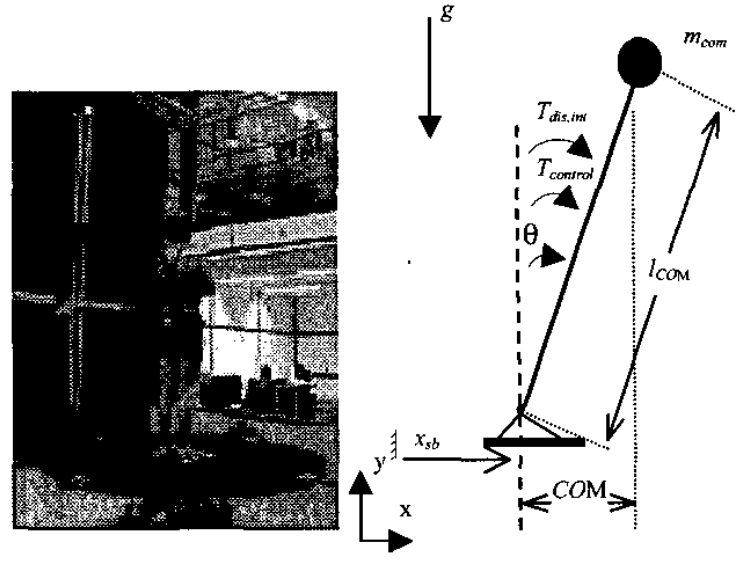

Figure 1. Experimental set-up (left) and inverted pendulum (right) as a simple model of the rigid body dynamics in standing. For used symbols see text.

frequency of $360 \mathrm{~Hz}$. From the ground reaction forces and moments the Center of Pressure (CoP) can be calculated, which is defined as the point of application of the ground reaction force on the force plate relatively to the ankle joint.

\section{Procedures}

In the present experiment a wide band multi-sine (WB) was applied to the forward-backward direction of the motion platform, resulting in continuous horizontal perturbations of the support base. The perturbation signals were multi-sines with specific harmonics and CREST optimized phases[9]. The task given to the participants was to keep upright with their arms folded in front of their chest, either with their eyes closed (EC) or open (EO). Both conditions were offered in a random order and each condition was repeated three times.

Before data recording, the participant was acquainted with the platform and the most difficult conditions. Prior to the start of the experiment the body posture was recorded in anatomical position. About $10 \mathrm{~s}$ after starting the perturbation signal the position of the markers was recorded for $65 \mathrm{~s}$. The time between two trials was about $25 \mathrm{~s}$.

\subsection{Parametric model of human balance control.}

A simple model of balance (Fig. 2.) is that of a human controller balancing an inverted pendulum on a movable platform with a mass equivalent with the body mass and with a length equivalent with the distance from the ankle joint to the (CoM). External disturbances and accelerations of the support base and internal disturbances such as breathing or movement of other body parts do destabilize posture. 


\section{$\underline{\text { Rigid Body Dynamics }}$}

The equations of motion of inverted pendulum on a movable platform can be obtained using Newton's laws. After linearisation of these equations around erect posture, they can be expressed in the frequency domain as:

$\theta(\omega, p)=P_{\text {ipend }}(\omega, p)\left[T_{\text {control }}(\omega)+w_{1}(\omega)+w_{2}(\omega)\right]$

In which $\omega$ is the angular velocity [ $\mathrm{rad} / \mathrm{s}], \theta$ is the body sway angle, $l_{\text {com }}$ is the distance from the CoM to the axis of rotation, $m_{\text {com }}$ is the body mass, $w_{l}$ are the internal perturbations, $w_{2}$ are external perturbations in this study evoked by accelerations of the support base $\left(\ddot{x}_{s b}\right)$, and $T_{\text {control }}$ is joint moment from the controller that stabilize posture (see later), and the transfer function of the inverted pendulum dynamics is:

$$
P_{i p e n d}(\omega, p)=\frac{1}{J(j \omega)^{2}-m_{c o m} g l_{c o m}}
$$

in which $J$ is moment of inertia and $g$ is gravitational acceleration. The mechanical effect of backwards (negative) accelerations of the support base times the CoM height and mass is equivalent with a joint moment around the ankle in forward direction (Fig. 2.).

The mechanical model is consistent with others [12, 13] but extended with the mechanical effect of support base translations and explicitly makes a distinction between internal and external perturbations. The reason why we explicitly distinguish internal $\left(w_{1}\right)$ and external $\left(w_{2}\right)$ perturbations is twofold: 1 ) external perturbations can be measured in contrast to internal perturbations; 2) internal perturbations do show up in CoP measurements while external perturbations and platform accelerations do not. This can be seen in the expression for the CoP of the linearised inverted pendulum model:

$C o P=-\frac{w_{1}+T_{\text {cantrol }}}{m_{\text {comm }} g}$

\section{Controller Dynamics}

The next step is a simple model for the human controller that has to stabilize the inherently unstable erect body posture. Position and velocity feedback results in positive stiffness and velocity feedback gains, needed to compensate for the negative stiffness due to gravity and to avoid oscillations, respectively. The intrinsic impedance of the muscles and visco-elastic tissues around the ankle joint and the neural position and velocity feedback are both possible sources for the required stiffness and velocity feedback gains. A general expression for the controller in which both types of feedback mechanisms are incorporated is:

$$
\begin{aligned}
& T_{\text {conrot }}(\omega, p)=K_{i} \theta(\omega)+D_{i} j \omega \theta(\omega)+\cdots \\
& \cdots+\left\{K_{r} \theta(\omega)+D_{,} j \omega \theta(\omega)\right\} e^{-\omega j t} H_{a c t}(\omega, p)= \\
& =\left[C_{l}(\omega, p)+C_{R}(\omega, p) H_{a c t}(\omega)\right] \theta(\omega)=C_{I R}(\omega, p) \theta(\omega)
\end{aligned}
$$

In this formula $K$ is the position feedback gain, $D$ is

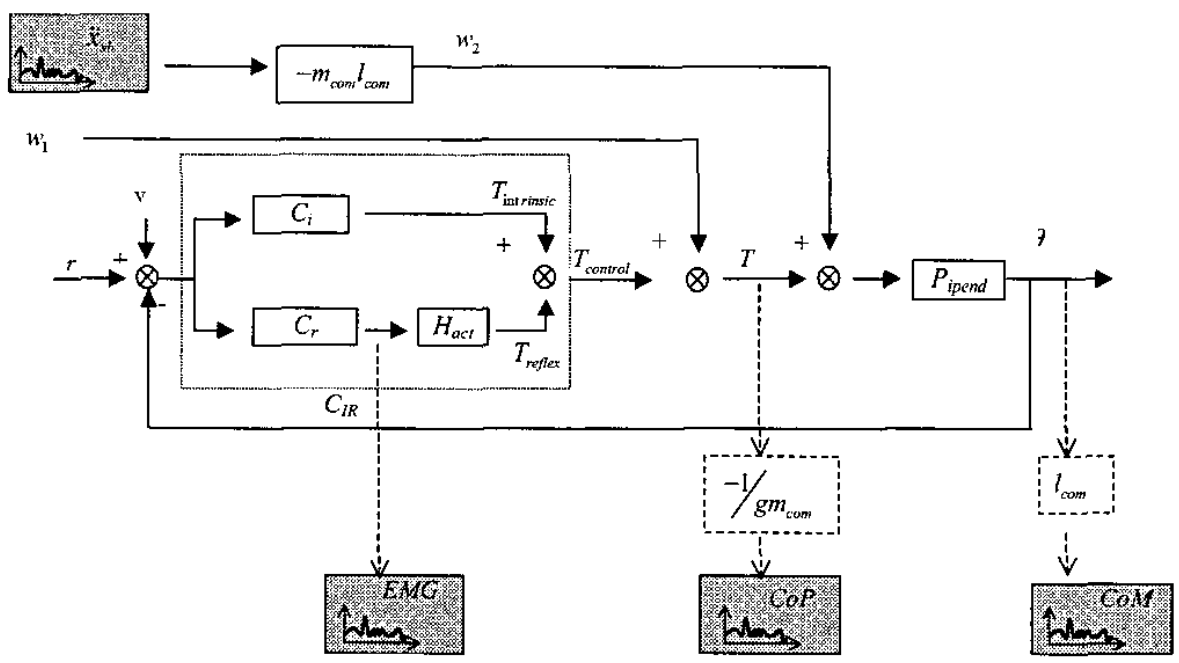

Figure 2

A simple model of human model control. $C_{I R}$ represents the dynamics of the human controller that consists of intrinsic $\left(C_{V}\right)$ and reflexive $\left(C_{R}\right)$ proportional and velocity feedback. $H_{a c t}$ represents the muscle activation dynamics, $P_{\text {ipend }}$ represents the dynamics of an inverted pendulum with the sum of control torque $\left(T_{\text {control }}\right)$ and internal $\left(w_{j}\right)$ and external $\left(w_{2}\right)$ perturbations as input and the body sway angle $(\theta)$ as output. The external perturbations are caused by platform accelerations $\left(\ddot{x}_{s b}\right), r$ is the reference or set point signal and $v$ represent unknown noise on the reference or in the feedback loop. The graph icons denote which signals can be measured measurements in balance control studies. 
the velocity feedback gain and $\tau$ is the lumped neural time delay. The subscripts $i$ and $r$ denote the intrinsic and reflexive contribution respectively. The used controller (4) is the same controller as used by $[14,15]$ but without the local Golgi Tendon force feedback loop. When force feedback is considered intrinsic and reflexive feedback are no longer independent [16].

The activation dynamics are described with a second order model[17-19]:

$$
H_{a c t}(\omega, p)=\frac{\omega_{o}^{2}}{(j \omega)^{2}+2 \beta \omega_{0} j \omega+\omega_{o}^{2}}
$$

in which $\beta$ is the relative damping and $\omega_{0}$ is the Eigenfrequency in $\mathrm{rad} / \mathrm{s}$ of the activation dynamics model.

In this paper we do assume that information about body sway angle and angular velocity is available. The problem of how humans are able to perceive their posture and motion in space is addressed elsewhere [20,21].

\section{Admittance}

The admittance or the sensitivity of the human balance control model to external perturbations $\left(w_{2}\right)$ is an important property that is a measure of how well the model is able to handle external perturbations:

$H_{n: 2 \theta}(\omega, p)=\frac{P_{i p e n d}(\omega, p)}{1+P_{i p e n d}(\omega, p) C_{I R}(\omega, p)}$

Although we can measure the $\mathrm{CoM}$, the $\mathrm{CoP}$ and the EMG of muscles involved in balance control there are also some properties that cannot be directly measured. We cannot measure the internal disturbances $\left(w_{l}\right)$. Although they show up in the CoP measurements they cannot be distinguished from the joint control torques $\left(T_{\text {control }}\right)$. Also the torques that are caused by the intrinsic mechanical properties $\left(T_{\text {intrinsic }}\right)$ of the muscular-skeletal system cannot be directly measured. Although the torques from the reflexive feedback pathways $\left(T_{\text {refiex }}\right)$ can be estimated from EMG recordings [22] of the major calf and pre tibial muscle groups they cannot be directly measured. Also the reference signal $(r)$ cannot be directly measured. The best guess is to assume that the reference corresponds with intention and task instruction. Fluctuations in the intention and the interpretation of the task can be modeled with a Gaussian noise (v) on the reference signal. Also sensory signal noise or other noise sources in the feedback loop can be modeled with this noise $v$. In the subsequent paragraphs we will call the noise $v$ the sensor noise although it captures both noise on the reference and within the feedback loop.

\subsection{Non-parametric closed-loop identify- cation of human balance control}

One of the major goals in studying human balance control is to identify the contribution and nature of intrinsic and reflexive feedback pathways in controlling balance in various environmental conditions. Since human balance control can be considered as a closed-loop system, closed-loop identification schemes have to be applied to identify the dynamics of the physiological control mechanisms.

The dynamics of the controller can be identified [2] by dividing the cross spectral densities of the external perturbation and CoP by the cross spectral densities of the external perturbations and the CoM

$\hat{C}_{I R}(\omega)=m_{C O M} l_{C O M} g \frac{\hat{\Phi}_{w 2 C O P}(\omega)}{\hat{\Phi}_{w 2 C O M}(\omega)} ; w_{1} \wedge w_{2} \neq 0$

The sensitivity to external perturbations or the admittance can also be estimated:

$\hat{S}_{\mathrm{w} 2}(\omega)=\frac{\hat{\Phi}_{w 2 \operatorname{COM}}(\omega)}{\hat{\Phi}_{w 2 w^{2}}(\omega)}$

To verify the usage of linear models the coherence function is estimated. This function is a measure of the linearity of the system. The estimated coherence function for the CoM signal is given by:

$\hat{\gamma}_{c o m}^{2}(\sigma)=\sqrt{\frac{\left|\hat{\Phi}_{k 2 c o m}(\sigma)\right|^{2}}{\hat{\Phi}_{x: 2 * 2}(\sigma) \hat{\Phi}_{\text {comcom }}(\sigma)}}$

In (6) and (7) the cross spectral density is used. Those spectral densities can be estimated from the product of the (complex conjugate) Fourier Coefficients $U$ and $Y$ from sampled time series data.

$\hat{\Phi}_{u j}^{N}(n \Delta \omega)=\frac{1}{N} U^{*}(n \Delta \omega) Y(n \Delta \omega)=\frac{1}{N} U(-n \Delta \omega) Y(n \Delta \omega)$

Those Coefficients are obtained with the Discrete Fourier Transform (DFT) of the time series $x$ of the sampled signals $u$ and $y$.

$X(n \Delta \omega)=\Delta t \sum_{k=0}^{N-1} x(k \Delta t) e^{-j 2 \pi n k / N} \quad$ voor $\mathrm{n}=0,1, \ldots \mathrm{N}-1$

in which $X$ and $x$ can be replaced with $U$ or $Y$ and $u$ or $y$. The signals $\mathrm{u}$ and $\mathrm{y}$ are sampled with a sample frequency $\left(f_{s}\right)$. The sample frequency $\left(f_{s}\right)$ and the 
observation time $(T)$ determine the frequency resolution $(\Delta \omega)$, time resolution $(\Delta t)$, and the number of samples $(N)$ :

$$
\Delta t=\frac{1}{f_{\mathrm{s}}} ; \Delta \omega=\frac{2 \pi}{T} ; N=f_{s} T
$$

The estimated gains, phases and coherences of all subjects and trials were averaged to obtain their means and standard deviations.

\subsection{Model parameter identification of intrinsic and reflexive properties}

The parameters for the inverted pendulum were obtained with an anthropometric scaling scheme [23]: $J=79 \mathrm{kgm}^{2} / \mathrm{s}^{2} ; l_{\text {com }}=1.05 \mathrm{~m} ; m_{\text {com }}=72 \mathrm{~kg}$. For the relative damping and the Eigenfrequency of the activation dynamics (5) values from [24] were used: $\beta=0.74$ and $\omega_{0}$ $=31.4 \mathrm{rad} / \mathrm{s}(5 \mathrm{~Hz})$. The other model parameters $\left(K_{i}, D_{i}\right.$, $\left.K_{n} D_{r}, \tau\right)$ were found by fitting the analytical expression for the dynamics of the human balance controller (4) on the corresponding mean estimated Frequency Response Function (FRF) (6). The unknown parameters were identified by minimizing the criterion function:

$$
L_{1}(p)=\sum_{k} \frac{\hat{\gamma}^{2}\left(\Phi_{k}\right)}{1+\varpi_{k}}\left|\ln \left(\hat{C}_{I R}\left(\Phi_{k}\right)\right)-\ln C_{I R}\left(\varpi_{k}, p\right)\right|^{2}
$$

For the minimization of (12) the FMINCON function of the MATLAB(@) optimization toolbox was used with defaults setting, except for the termination tolerance that was 1 e- 62 based on the function value. The optimization was repeated ten times with different initial settings. Local optimums were removed. The means and standard deviations of the found parameters were calculated.

\section{Model validation:}

The variance accounted for (VAF) in the frequency domain is calculated to obtain an index for 'the goodness of fit' of the model predicted to the estimated FRF's of the controller dynamics.

$V A F_{C I R}=\left[1-\frac{\sum_{1}^{N}\left|\hat{C}_{I R}\left(\omega_{k}\right)-C_{I R}\left(\omega_{k}, p\right)\right|^{2}}{\sum_{1}^{N}\left|\hat{C}_{I R}\left(\omega_{k}\right)\right|^{2}}\right] \times 100 \%$

\section{Results}

\subsection{Nonparametric FRF's}

In the mean estimated FRF's of the scaled controller dynamics $\left(C_{I R} / m_{\text {com }} g l_{\text {com }}\right)$ the effect of velocity feedback can be recognized as the phase lead at lower frequencies and the effect of the neural time delay as the phase lag at higher frequencies (Fig. 3). The system is critically damped or over-damped since in the estimated admittance no resonance peak is observed. From the estimated admittance at lower frequencies it appears that the balance control system is characterized by a very low effective stiffness since the gain of the scaled controller dynamics is about one. Since the estimated FRF of the controller dynamics is scaled by $m_{\text {com }} g l_{\text {com }}$ the consequence is that the controller torque is just a little larger than the toppling torque $\left(m_{\text {com }} g l_{\text {com }} \theta\right)$ ). In general the coherence of the estimated transfer function is high, with the exception of the coherence of the FRF's at lowest frequency for the EC condition. The estimated gain of the scaled FRF of the controller dynamics is smaller than one for this situation, resulting in a unstable system, but can be considered as an unreliable estimate since the coherence is very small.

\subsection{Intrinsic and reflexive model parameters}

The unknown model parameters were found by repeated optimization (Table 2). The model accounted for more than $98 \%$ of the variance of the estimated FRF's of the dynamics of the controller (Table 2). The gain and phase of the model and the estimated FRF of $C_{I R}$ did correspond well, with exception of the gain at the lowest frequency for EC condition (Fig 3). The model predicted and estimated FRF's of the admittance also correspond well, although this FRF was not used in the fitting procedure. With the used termination toleration of the optimization procedure small variations in the object function $\left(L_{1}(p)\right)$ and in the model parameters were found as reflected by the standard deviations (Table 2). The differences in the found solutions were small and could not be recognized by visual inspection of the different corresponding FRF's. Lowering the termination tolerance of the optimization routine reduced the variation in the model parameters (not shown). Consistent solutions were found for the intrinsic damping and neural velocity feedback gain and time delay. However, no consistent solutions were found for the intrinsic stiffness and the neural position feedback gain. However, the sum of both was always the same for various initial parameter values in the model fit optimization procedure. The sum of the intrinsic and reflexive position feedback is always larger than the gravitational stiffness, $m_{c o m} g l_{\text {com }} \sim 741 \mathrm{Nm} / \mathrm{rad}$, which is a necessary condition for stability. For EC condition the estimated sum of $K_{i}$ and $K_{r}$ and the estimated $D_{r}$ was higher than in EO condition. 


\section{Discussion}

The high VAF in combination with the high estimated coherences suggests that a linear model with position and velocity intrinsic and delayed feedback pathways is an accurate model to describe the mechanical effects of horizontal platform accelerations on human body sway. In comparison with others (Table 1) we do not use erroneous open-loop identification techniques. Other researchers that used proper closed-loop identification techniques $[8,10]$ only estimated the admittance and did not isolate the controller dynamics as we did.

Estimating and fitting the FRF's of the controller dynamics from $\mathrm{CoM}$ and $\mathrm{CoP}$ data resulted in unique values for the neural time delay and velocity feedback gains and the intrinsic damping but not for the intrinsic and neural position feedback gains. To distinguish between the intrinsic stiffness and the neural position feedback the closed-loop FRF's of the CoM to the neural activation should be estimated [2] as in [24] was done for the arm. The estimated velocity feedback can be mainly attributed to neural feedback rather than intrinsic feedback. The very low intrinsic damping is in

\begin{tabular}{l|ll} 
& \multicolumn{2}{c}{ Table 2 } \\
& \multicolumn{2}{c}{ Identified Model Parameters } \\
& EO L $_{1}$ & EC L $_{1}$ \\
\hline$\left.K_{i}+K, / \mathrm{Nm} / \mathrm{rad}\right)$ & $742 \pm 1$ & $835 \pm 5$ \\
$\left.D_{i} / \mathrm{Nms} / \mathrm{rad}\right]$ & $0.1 \pm 0.1$ & 0 \\
$\left.D_{r} / \mathrm{Nms} / \mathrm{rad}\right]$ & $122 \pm 12$ & $194 \pm 2$ \\
$\tau[\mathrm{ms}]$ & $66 \pm 6$ & $59 \pm 7$ \\
$L(p) /]$ & $0.032 \pm 0.001$ & $0.024 \pm 0.003$ \\
$V A F / \%]$ & $98.6 \pm 0.2 \%$ & $98.2 \pm 0.4 \%$
\end{tabular}

correspondence with [5] and[3].

The model fit procedure is sensitive for the used criterion and for the assumed activation dynamics (results not shown), which needs further investigation.

\section{Conclusions}

Multi sinus horizontal platform perturbations evoke postural responses in standing human subjects with EO or EC that could be used to obtain reliable estimates of the FRF's of the physiological balance control mechanisms. The found FRF's could be fitted with a simple balance control model. The model fit resulted in consistent estimates of the sum of the intrinsic stiffness and the reflexive position feedback, the intrinsic damping, the
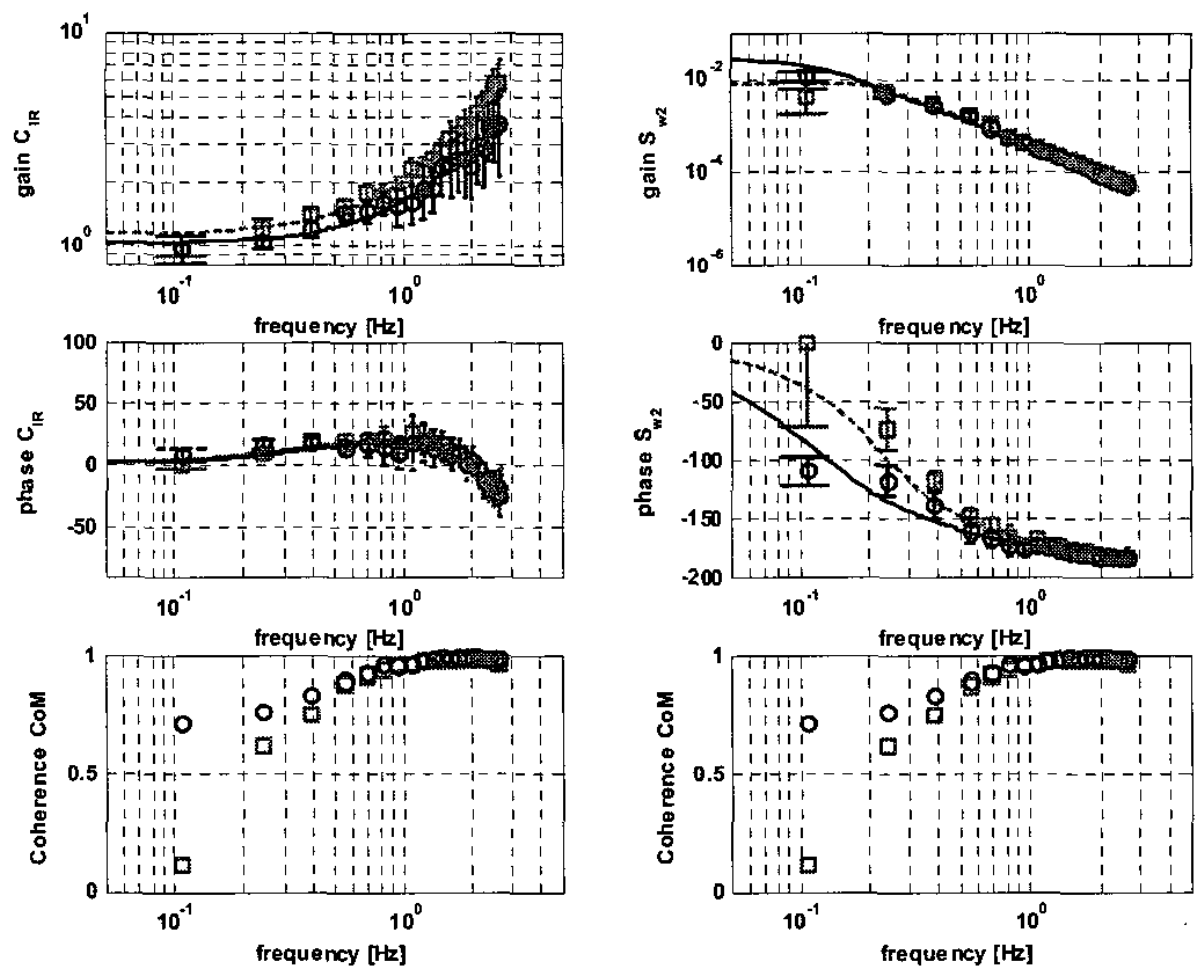

Fig.3: Mean and standard deviations of gain (top panels) and phase (middle panels) and coherence (bottom panels) of the estimated FRF's (EO circles: EC squares) of the controller dynamics $C_{I R}$ scaled by $m_{\text {com }} g l_{\text {com }}$ (left panels) and the estimated admittance ((EO circles: EC squares). Error bars denote the $95 \%$ confidence intervals of the estimates. The model fitted FRF's are also shown for EC (dotted lines) and EO (solid lines). Note that boxes and circles are the estimated FRF's and the lines the fitted model FRF's curves. 
reflexive velocity feedback gains and the neutral time delay of the reflexive feedback pathway. The fitted model parameters suggests that: 1) subject applied a minimal stiffness strategy: 2) position and velocity feedback is larger for EC than for EO; 3) velocity feedback is dominated by reflexive feedback and not by intrinsic damping.

\section{References}

[1] I. D. Loram, S. M. Kelly, and M. Lakie, "Human balancing of an inverted pendulum: is sway size controlled by ankle impedance?," J Physiol, vol. 532, pp. 879-91, 2001.

[2] H. v. d. H. van der kooij, F.C.T.; "The pitfalls of using (open-loop) identification techniques in balance control studies: the cause of controversies and speculations.," Journal of Neuroscience Methods, submitted.

[3] I. D. Loram and M. Lakie, "Direct measurement of human ankle stiffness during quiet standing: the intrinsic mechanical stiffness is insufficient for stability," J Physiol, vol. 545, pp. 1041-53, 2002.

[4] I. D. Loram and M. Lakie, "Human balancing of an inverted pendulum: position control by small, ballistic-like, throw and catch movements," $J$ Physiol, vol. 540, pp. 1111-24, 2002.

[5] M. M. Mirbagheri, H. Barbeau, and R. E. Kearney, "Intrinsic and reflex contributions to human ankle stiffness: variation with activation level and position," Experimental Brain Research, vol. 135, pp. 423-36, 2000.

[6] B. Koopman, H. J. Grootenboer, and H. J. de Jongh, "An inverse dynamics model for the analysis, reconstruction and prediction of bipedal walking," J Biomech, vol. 28, pp. 1369-76, 1995.

[7] M. Mihelj, Z. Matjacic, and T. Bajd, "Postural activity of constrained subject in response to disturbance in sagittal plane," Gait Posture, vol. 12, pp. 94-104, 2000.

[8] R. J. Peterka, "Sensorimotor Integration in Human Postural Control," Journal of Neurophysiology, vol. 88, pp. 1097-1118, 2002.

[9] G. P. Schoukens J, Pintelon R, "Design of broadband excitation signals," in Perturbation Signals for System Identification, G. KR, Ed. Englewood Cliffs, USA: PrenticeHall, 1993.

[10] T. Sinha and B. E. Maki, "Effect of forward lean on postural ankle dynamics," IEEE Trans Rehabil Eng, vol. 4, pp. 348-59, 1996.

[11] R. C. Fitzpatrick, J. L. Taylor, and D. I. McCloskey, "Ankle stiffness of standing humans in response to imperceptible perturbation: reflex and task-dependent components," J Physiol, vol. 454, pp. 533-47, 1992.
[12] R. J. Peterka, "Postural control model interpretation of stabilogram diffusion analysis.," Biological Cybernetics, vol. 82, pp. 335-343, 2000.

[13] D. A. Winter, A. E. Patla, F. Prince, M. G. Ishac, and K. Gielo-Perczak, "Stiffness control of balance in quiet standing.," Journal of Neurophysiology, vol. 80, pp. 1211-1221, 1998.

[14] F. C. van der Helm, A. C. Schouten, E. de Vlugt, and G. G. Brouwn, "Identification of intrinsic and reflexive components of human arm dynamics during postural control," J Neurosci Methods, vol. 119, pp. 1-14, 2002.

[15] A. J. van Soest, W. P. Haenen, and L. A. Rozendaal, "Stability of bipedal stance:the contribution of cocontraction and spindle feedback.," Biological Cybernetics, vol. 88, pp. $293-301,2003$.

[16] F. C. T. van der Helm, Rozendaal, L.A., "Musculoskeletal systems with intrinsic and proprioceptive feedback," in Neural control of posture and movement, J. M. Winter, Crago, P, Ed. New York: Springer Verlag, 2000, pp. 164174.

[17] S. J. Olney and D. A. Winter, "Predictions of knee and ankle moments of force in walking from EMG and kinematic data," J Biomech, vol. 18, pp. 9-20, 1985.

[18] J. Bobet and R. W. Norman, "Least-squares identification of the dynamic relation between the electromyogram and joint moment," J Biomech, vol. 23, pp. 1275-6, 1990.

[19] J. R. Potvin, R. W. Norman, and S. M. McGill, "Mechanically corrected EMG for the continuous estimation of erector spinae muscle loading during repetitive lifting," Eur J Appl Physiol Occup Physiol, vol. 74, pp. 119-32, 1996.

[20] H. van der Kooij, R. Jacobs, B. Koopman, and H. Grootenboer, "A multisensory integration model of human stance control," Biological Cybernetics, vol. 80, pp. 299-308, 1999.

[21] H. van der Kooij, R. Jacobs, B. Koopman, and F. van der Helm, "An adaptive model of sensory integration in a dynamic environment applied to stance control," Biological Cybernetics, vol. 84 , pp. 103-115, 2001.

[22] D. G. Lloyd and T. F. Besier, "An EMG-driven musculoskeletal model to estimate muscle forces and knee joint moments in vivo," J Biomech, vol. 36, pp. 765-76, 2003.

[23] Y. C. Pai and J. Patton, "Center of mass velocityposition predictions for balance control," Journal of Biomechanics, vol. 30, pp. 347-54, 1997.

[24] A. C. d. V. Schouten, E.;van Hilten, J. J.;van der Helm, F. C.T., "Quantifying spinal reflexes in vivo during postural control," Journal of Neuroscience Methods, submitted. 